\title{
Terminal
}

Technologie de l'information, culture \& société

$123 \mid 2018$

Haine et violence numérique

\section{Un usage déviant du numérique : le cas des détenteurs et des diffuseurs de vidéos pédopornographiques sur internet}

Frédéric Pugniere-Saavedra

\author{
(2) OpenEdition \\ Journals \\ Édition électronique \\ URL : http://journals.openedition.org/terminal/3317 \\ ISSN : 2429-4578 \\ Éditeur \\ CREIS-Terminal
}

Ce document a été généré automatiquement le 25 février 2019. 


\title{
Un usage déviant du numérique : le cas des détenteurs et des diffuseurs de vidéos pédopornographiques sur internet
}

\author{
Frédéric Pugniere-Saavedra
}

1 Nous considérons dans cette contribution que le cyberespace est moins un espace où règne la haine et/ou la violence numérique qu'un espace qui facilite et accélère l'accès à des données qui véhiculent des comportements déviants. Nous prenons d'abord appui sur la définition proposée par Douzet (2014) pour qui le cyberespace, « c'est à la fois l'Internet et l'« espace » qu'il génère : un espace intangible dans lequel s'opèrent des échanges déterritorialisés entre des citoyens de toutes nations, à une vitesse instantanée qui abolit toute notion de distance » (p.6) pour la dépasser en ajoutant que le cyberespace est un territoire sans périmètre qui renvoie à des imaginaires flous avec ses zones de non-droit.

2 Notre analyse portera sur un corpus de récits de vie recueillis dans le cadre de groupes de paroles auprès de consommateurs et de diffuseurs de vidéos pédopornographiques entre 2015 et 2017. Nous nous intéresserons ici à la mise en mots de leur histoire familiale, de leurs valeurs, de leurs croyances et de leurs propres trajectoires en lien avec la technologie et les enfants mineurs. À travers ce type de délinquants sexuels, nous réfléchirons à la figure de l'Autre, qui est un enfant dans une relation à distance via la technologie numérique et connectée. Autrement dit, la technologie reconfigure-t-elle les émotions et les actes à destination des enfants? La technologie désincarne-t-elle l'enfant? L'omniprésence des écrans et l'accès multimodal facilité par internet impliquet-il une reconfiguration des pulsions? Comment penser la place du virtuel et le questionnement pathologique que soulève ce dispositif technique? Comment le consommateur de pédopornographie catégorise-t-il l'enfant, comment le met-il en mots? Comment apparait dans leur discours la notion de responsabilité ? Telles seront les interrogations qui jalonneront cette réflexion autour de ce corpus réduit et exploratoire. 


\section{Éléments de cadrage}

3 L'analyse prendra appui sur deux champs théoriques: d'une part celui des sciences de l'information et de la communication en privilégiant la conception d'une société dite numérique (Zetlaoui 2016, Boullier 2016, travaux du CREIS, Terminal, 110|2012) caractérisée par la pluralité des formes que prennent les usages (Chambat 1994) allant de "l'adoption" à "l'appropriation" en passant par "l'utilisation" (Breton et Proulx) «l'appropriation de la technique exige, selon Proulx et Breton, la réunion de trois conditions sociales. Pour s'approprier un objet technique, l'individu doit en effet démontrer un minimum de maîtrise technique et cognitive de cet outil. Cette maitrise devra s'incorporer de manière créatrice à ses pratiques courantes. Par ailleurs, l'appropriation doit pouvoir donner lieu à des possibilités de détournements, de réinventions, voire de contributions directes des usagers à la conception des innovations techniques. Cette définition pose à la fois le cadre à l'intérieur duquel les locuteurs pensent l'outil, l'utilisent et s'expriment par et à travers lui » (2002: 244). Nous considérons que cette société numérique forme un écosystème entendu comme l'ensemble des équipements permettant le passage à Internet et l'ensemble des services associés quel que soit le secteur ; nous situerons davantage notre propos dans la finalité des données produites et des usages du numérique par les détenteurs et les diffuseurs de vidéos pédopornographiques.

4 D'autre part, celui des sciences du langage mobilisant le discours tel que défini par Paveau: "Les discours ne sont pas seulement des suites de mots et de phrases, mais constituent des lieux où se définissent les normes et les valeurs, les prescriptions et les interdits, les goûts et les dégoûts, les qualités et les défauts, les identités, les légitimités, les gloires et les hontes» (2014: 25). Les productions discursives des locuteurs seront appréhendées à la fois sous le prisme de l'analyse de discours (tendance française) qui prend appui sur les marques d'énonciation et qui considère qu'il existe des invariants, des propriétés spécifiques linguistiques et discursives pour rendre observables des configurations spécifiques de ce type de récits. Elles seront également appréhendées sous le prise d'une perspective élargie ${ }^{1}$ de la définition de l'argumentation défendue par Amossy (2000: 37$)$ pour qui «les moyens verbaux qu'une instance de locution met en œuvre pour agir sur son allocutaire en tentant de la faire adhérer à une thèse, de modifier ou de renforcer les représentations et les opinions qu'elle leur prête, ou simplement d'orienter leurs façons de voir ou de susciter un questionnement sur un problème donné $»^{2}$. Transversalement, ces productions discursives seront appréhendées par une approche textométrique qui propose une approche instrumentée des corpus, articulant synthèses quantitatives et analyses à même le texte (Lebart \& Salem 1994, Mayaffre 2007).

\section{Présentation du corpus de travail}

5 Pour cette contribution, nous n'avons retenu que les huit récits de vie de consommateurs et de diffuseurs de vidéos pédopornographiques ayant participé à ces groupes de paroles ${ }^{3}$. Il ne s'agit que de récits de vie d'hommes jeunes (moins de trente ans) qui se trouvent en position de justification de leurs actes. 


\begin{tabular}{|l|l|}
\hline $\begin{array}{l}\text { Locuteur anonymé } \\
4\end{array}$ & Faits marquants \\
\hline Louis & n'a pas été inquiété par la police au moment du groupe de parole \\
\hline Jules & En cours de jugement au moment du groupe de parole \\
\hline Alain & $\begin{array}{l}\text { En cours de jugement et en cours de traitement pour mettre fin aux } \\
\text { addictions }\end{array}$ \\
\hline Albert & 25 années d'addiction, en attente de jugement \\
\hline Tom & Saisie du matériel, en cours de jugement \\
\hline Edouard & Condamné à suivre une thérapie \\
\hline Marc & Peine purgée il y a 2 ans \\
\hline Thomas & En cours de jugement au moment du groupe de parole \\
\hline
\end{tabular}

6 Ces récits de vie tels que définis par Bertaux (1997: 35) « dès lors qu'un sujet raconte à quelqu'un d'autre un épisode quelconque de son expérience de vécue» [...] "pour raconter une histoire, il faut camper des personnages, décrire leurs relations réciproques, expliquer leurs raisons d'agir; décrire les contextes des actions et interactions", sont donc légitimes en tant qu'ils émanent d'un locuteur socialement impliqué dans son dire.

7 Cette définition est complétée dans son versant linguistique (Nossik 2011) en tant «qu'activités (re)configurant les réalités sociales vécues des locuteurs par le choix des constructions singulières de la syntaxe, du lexique et de formes énonciatives ». Ces récits de vie de consommateurs de vidéos pédo-pornographiques prennent forme au travers de groupes de paroles (Bass et Caevel 2005) en tant que «scène d'expression langagière et d'écoute qui doit être interrogée sur les effets réflexifs de chaque participant. Ces récits de vie constituent par ailleurs un corpus dit sensible (Paveau et Pérea 2012/2015) car il ne figure pas dans les habitus institutionnalisés de la recherche et se manifeste principalement par sa charge d'interdits, ce qui questionne nécessairement le chercheur par rapport à sa pratique et à son objet.

Deux focales seront donc abordées pour fournir des éléments de réponse aux questions supra: une première focale portant sur la question de la responsabilité des consommateurs et des diffuseurs de vidéos pédopornographiques et plus spécifiquement, sur la minoration de leurs responsabilités (3.1), une seconde focale portant sur la manière dont les auteurs mettent en place des mécanismes de défense qui reconfigurent le réel (3.2).

\section{Analyses}

9 Les locuteurs mobilisent les phénomènes de désignation (Siblot 1998, Branca-Rosoff 2007) pour parler d'eux-mêmes, pour évoquer leurs actes, leurs victimes et leur dépendance à l'outil numérique. Les désignations du consommateur de vidéo ( $\mathrm{cf}$. tableau $\mathrm{n}^{\circ} 1$ ) se 
répartissent entre hyperonyme (agresseur) et hyponymes (pédophile, délinquant sexuel, abuseur) objectifs et désignations subjectives (monstre, cybercriminel, criminel).

Tableau $\mathrm{N}^{\circ} 1$ : désignations de 3 champs lexicaux

\begin{tabular}{|l|l|}
\hline $\begin{array}{l}\text { Nature du } \\
\text { champ lexical }\end{array}$ & Désignations se rapportant au champ lexical \\
\hline technologie & $\begin{array}{l}\text { Peer-to-peer [17], ordinateur [17], Internet [16], virtuel [10], site de } \\
\text { téchargements [9], tchat(s) [6], fichiers [6], informatique [5], sms [4], adresse } \\
\text { algorithme [1], newsgroup [1], minitel [1]. }\end{array}$ \\
\hline agresseur & $\begin{array}{l}\text { Pédophile(s) [18], prédateur [11], criminel [10] délinquant sexuel [9], Monstre } \\
{[9], \text { abuseur [6], cybercriminel [5]. }}\end{array}$ \\
\hline enfant & $\begin{array}{l}\text { Enfant(s) [71], fille (s) [63], petite fille [31], jeune(s) fille(s) [28], gamin(s) [16], } \\
\text { gamine(s) [12], victime(s) [11], garçon(s) [9], gosse [3], mineur [3], fillette(s) [2]. }\end{array}$ \\
\hline
\end{tabular}

\section{Entretien Louis}

Et moi je me voyais comme un monstre, je me voyais comme le monstre qui était mon grand-père... La seule personne qui m'a aimé c'était lui, et en fait c'était un monstre

Certaines désignations du mineur sont objectives (jeune-fille, garçon, petite fille, jeune garçon, victime) d'autres plus subjectives (gamin(e), fillette).

\section{Entretien Jules}

En dernière page de la revue, il y avait une petite fille qui était nue et moi, pendant longtemps, je pensais que c'était ça qui m'avait un peu incité à aller rechercher ben les caractères sexuels pédopornographiques.

11 D'autres procédés de désignations sont mobilisés pour construire une figure singulière en termes de responsabilités du consommateur de vidéos à caractère pédo-pornographique.

\section{Mise en mots pour minorer la responsabilité des auteurs}

12 Les participants optent pour ne pas nommer explicitement l'acte en recourant à des coréférents qui renvoient volontairement à un espace temporel vague (Brunner 2014) sans bornes de début ou de fin (cf. tableau $\mathrm{n}^{\circ} 2$ ) sauf quand il y eu garde à vue où la date et l'heure sont restées en mémoire puisque cet élément constitue un point référentiel dans l'histoire du participant.

\section{Entretien Alain}

Deuxième garde à vue, euh, deuxième réveil à 6 heures du mat', tambourinage à ma porte, enfin plutôt à ma sonnette puisque ma porte n'est pas directe accessible

Tableau $N^{\circ} 2$ : désignations de la temporalité

Désignations se rapportant à la temporalité 
Age : jeune adulte, 10 ans, 13 ans

Date/heures : le 6 novembre, à $6 \mathrm{~h}$ du mat', à 2 heures du matin, à $5 \mathrm{~h}$ du mat'

Connecteurs énonciatifs : 3 jours plus tard, le jour où, le premier jour de, à cette époque-là, à ce moment-là de ma vie.

Durée : condamné à 4 mois

13 Ces co-référents sont construits avec un déictique (mon histoire, cet évènement, dans mon souvenir) (tableau 3, exemples 1 et 2), avec des désignations abstraites omnisignifiantes (truc) ou des pronoms cataphoriques (ça).

Tableau N³ :désignations de l'acte avec ses co-référents

désignations de l'acte avec ses co-référents

ça (513), truc (35), cet événement (7), acte (7), histoire (5), souvenir (4).

\section{Exemples 1}

\section{Entretien Jules}

Donc c'était, c'était ça un peu, c'était compliqué, je me rappelle, à cette époque-là, euh mon développement, je sais pas, à l'adolescence, j'étais perturbé

Entretien Alain

Je veux dire, en fait notre relation n'a pas changé de ce qu'elle était avant, avant cet événement. Donc à partir de ce moment-là j'ai vraiment pu faire un vrai travail qui maintenant m'a...aidé.

Entretien Albert

Je vais revenir un peu sur le téléchargement, sur mon histoire.

\section{Exemple 2}

\section{Entretien Alain}

Après euh, après beaucoup de travail, euh, avec euh, avec le groupe de parole et puis avec le psy chez qui j'ai été pendant quelques années, euh, j'ai jamais réussi réellement à déterminer le point de départ, en fait, de ma pédophilie, de quoi ça venait, euh, à quoi c'était dû, euh, je n'ai pas eu une enfance perturbée, j'ai pas été victime d'abus sexuel, j'ai toujours été dans un foyer chouaillé on va dire, j'ai pas eu de problème à ce niveau-là. Je ne peux pas, je n'ai pas de point de focalisateur qui me permettrait de dire à tel moment il y a eu un problème dans ma vie, et euh, à partir d'un moment, ça a dévié, euh, ça je ne peux pas dire, je sais que d'assez loin où j'ai réussi à retourner dans le passé, j'ai tout le temps eu une attirance effectivement pour euh, les jeunes filles pas pour les petites filles mais pour les jeunes filles.

On constate qu'il y a un tiraillement dans le processus de désignation. Dans leur récit, les participants ne se perçoivent pas tous comme "pédophiles » (exemple $\mathrm{n}^{\circ} 3$ détaillé plus loin), ils ont peu conscience de l'élément déviant déclencheur, expliquent peu leur évolution personnelle. Pour cela, ils mobilisent des désignations englobantes pour monter en généralité et amoindrir de ce fait leurs responsabilités. 


\section{Exemple 3}

\section{Entretien Albert}

Je n'y avais pas été parce que pour moi je ne suis pas pédophile enfin au sens, enfin

je ne suis pas pédo sexuel, enfin je veux dire, enfin un enfant c'est juste pas possible

Ce tiraillement entre raconter son histoire singulière dans cette situation particulière de discours et être compris des autres participants ${ }^{5}$ mobilise un travail d'ajustement de la désignation par rapport au monde où «la référence n'est que l'état résultatif d'un processus de référenciation, qui ne se fonde pas sur une conception objective du rapport langage/monde, mais sur la médiation sémiotique des ressources langagières dans la mise en mots du réel selon un certain point de vue» (Longhi 2015: 131). Ce travail d'ajustement lexical se manifeste également dans la manière de nommer les outils technologiques et l'enfant. En effet les participants se limitent à nommer le support (photo, image, film) pour évoquer l'enfant, comme si ce dernier était désincarné dans la séquence où le participant présente les faits.

Figure 1 : répartition des outils technologiques par participant

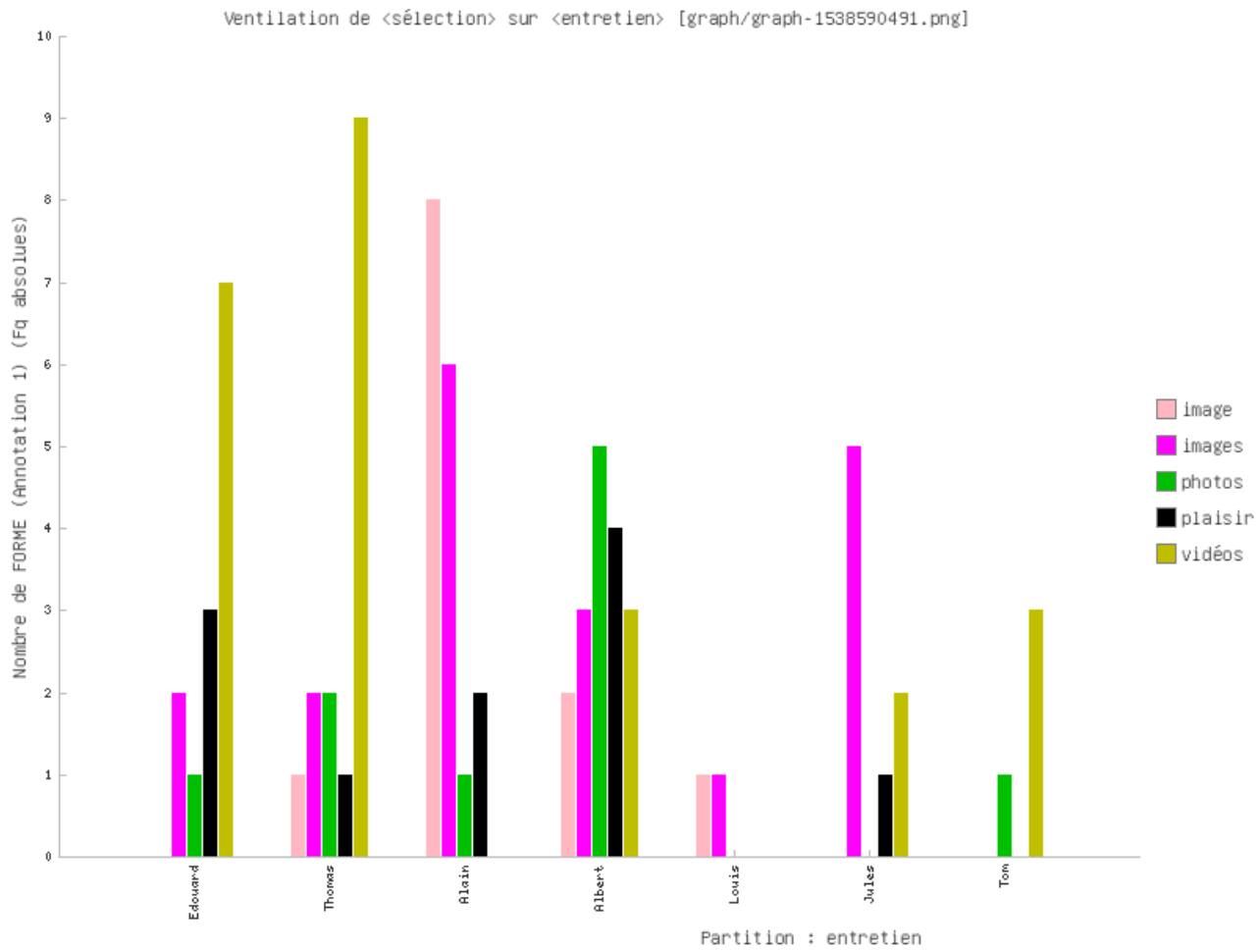

\section{Exemples $n^{\circ} 4$}

\section{Entretien Alain}

Locuteur Alain

J'entame, euh, donc je m'appelle Alain, j'ai 40 ans et euh, j'ai donc été reconnu coupable de téléchargements importation, exportation et tout le toutim de ça... d'images et de film donc les faits initiaux ont maintenant une dizaine d'années. J'ai été deux fois en procès et voilà...

\section{Entretien Louis}

Je suis devenu effectivement accro à ça [la pédopornographie], je ne me suis jamais fait attraper. J'ai arrêté plusieurs fois les téléchargements d'images vidéo. J'ai arrêté déjà depuis déjà quand même assez longtemps, ça se compte en ... quelques 
années quoi. Je télécharge aussi des dessins et il se trouve que légalement maintenant les dessins aussi sont illégaux.

16

\section{Entretien Jules}

Parce que quand on va sur Internet, sur certaines pages, on cherche des dessins, et puis entre deux images de dessins, et ben il y a une photo qui est vraie, et donc du coup ben... ouais, je sais pas. Peut-être, ouais. Je sais pas trop en fait comment j'ai pu en arriver là, j'ai des... ouais, je pense que j'ai des... suppositions, mais je peux pas affirmer avec certitude que... c'est par exemple la fois où c'est que je me suis masturbé devant ce dessin animé que... Enfin devant ce cahier qui contenait des dessins que... qui a fait que... que...

\section{Entretien Alain}

Locuteur Alain Ouais, je me suis r'trouvé dans des situations où effectivement, si j'avais été un monstre, j'aurais été plus loin à ce moment-là et puis finalement j'ai rien fait, c'est pas très grave, c'était que des images...

\section{Entretien Thomas}

Locuteur Thomas

Ben, pour commencer, je suis un... je pense, « cyber-péd-pédophile », c'est comme ça qu'on dit ? Je consomme des images sur Internet. J'ai commencé ça il y a très longtemps, j'avais... treize ans à peu près.

[...]

Locuteur Marc (victime qui a été filmé en train de se faire caresser les parties génitales dans les douches par un surveillant en colonie de vacances alors qu'il avait 8 ans)

Alors on peut pas dire «image» Thomas. Parce que c'est des enfants. Tu comprends? Parce que quand on dit «image», c'est vrai qu'on a une autre connotation. Hein? On a l'impression qu'on est en train de regarder une image. Alors que derrière cette image d'enfants... Faut mettre des mots sur des mots

Et plus tard au cours du groupe de paroles un autre participant rectifie de lui-même la nomination

\section{Exemples $\mathrm{n}^{\circ} 5$}

\section{Entretien Tom}

Locuteur Tom

Donc euh... Moi j'ai commencé, euh... comme monsieur, par euh... des téléchargements d'i... d'images ... d'enfants et de vidéos. Par contre, contrairement à monsieur...

Modératrice

Vous voyez il dit : « enfants », « images d'enfants »! (rires)

Parallèlement à ces constructions désignationnelles particulières, les participants mettent largement en avant ce qui ne va pas et la recherche de compréhension de ce qui a pu les mener à la pédopornographie pour valoriser leur distorsion de la réalité.

Structurellement, ces énoncés sont construits négativement avec des atténuateurs (restrictifs, oppositifs) propres à l'oral («je ne suis pas, je ne sais pas, je ne peux pas.... » + atténuateurs enfin, je veux dire, mais...). Les quatre premiers adverbes les plus fréquents par ordre décroissants ont une valeur sémantique négative (cf. tableau $n^{\circ} 4$ ) et les constructions les plus fréquentes (par ordre décroissant) sont les suivantes : Je ne sais pas (19 occurrences), je ne suis pas (17 occurrences) et je ne peux pas (13 occurrences); l'occurrence suivante je ne vais pas descend à 4 occurrences : (cf. figure $\left.\mathrm{n}^{\circ} 2\right)$. 


\begin{tabular}{|l|l|}
\hline Fréquence & Terme \\
\hline 673 & pas \\
\hline 181 & plus \\
\hline 179 & ne \\
\hline 175 & Non \\
\hline 144 & là \\
\hline 106 & même \\
\hline 101 & donc \\
\hline
\end{tabular}

Figure 2 : répartition du patron Adv+verbe au pres+adv

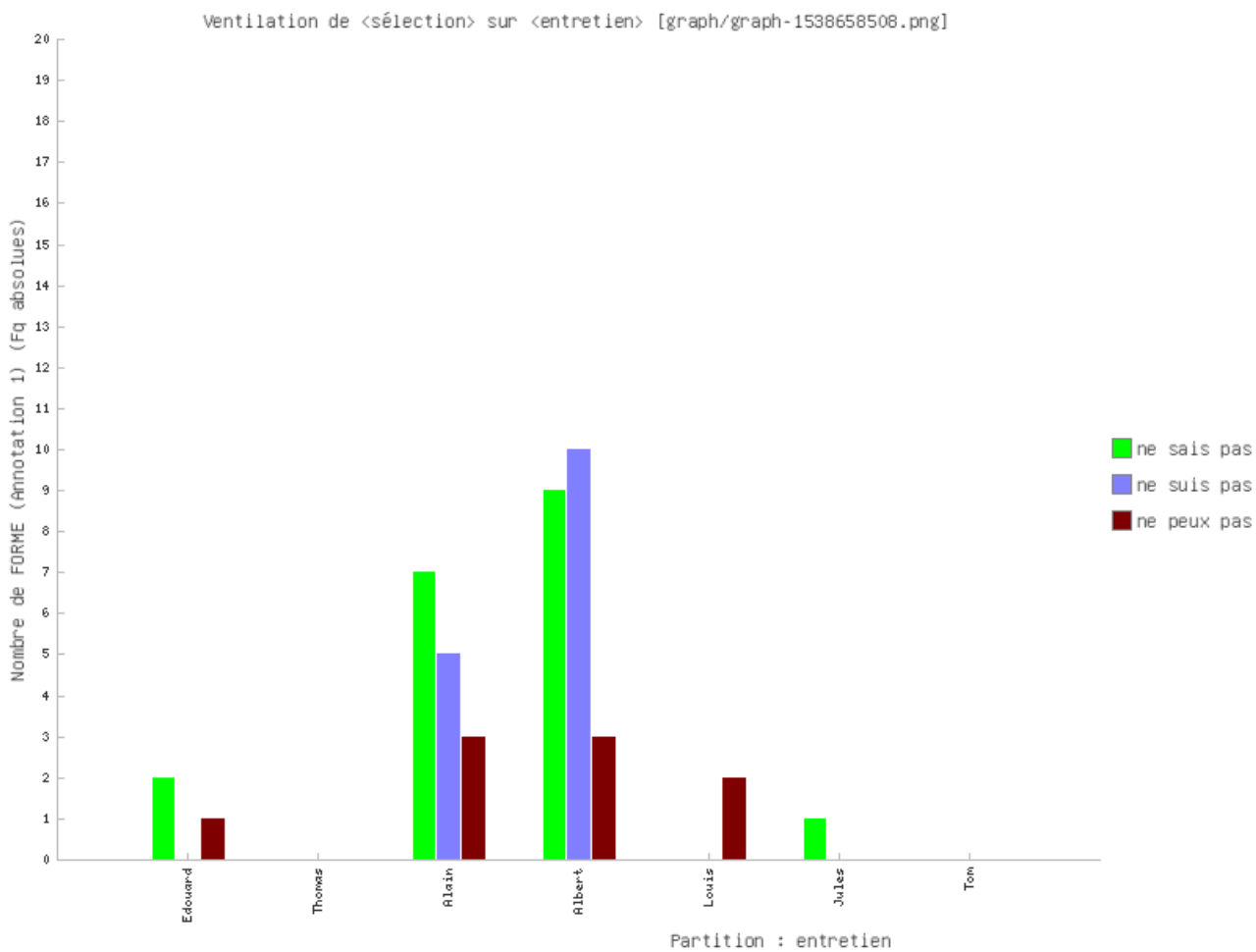

Entretien Albert

Moi je ne supporte pas l'image de «vous êtes pédophile», je ne suis pas pédophile je suis en train de revivre des cauchemars.

Je n'y avais pas été parce que pour moi je ne suis pas pédophile enfin au sens, enfin je ne suis pas pédo sexuel, enfin je veux dire, enfin un enfant c'est juste pas possible Je ne sais pas encore. J'y travaille. Je travaille dessus, j'essaie d'analyser ça mais c'est pas une démarche difficile de...

Ces amorces négatives se co-construisent avec des énoncés qui renvoient à une forme de compulsion et in fine de plaisir. Les désignations des supports (image, film, vidéo) subissent une actualisation quantitative (exemples $n^{\circ} 5$ ) qui provoque une forme de distorsion dans le réel conduisant le locuteur à la fois à exprimer des troubles (exemple $n$ ${ }^{\circ} 6$ ) et à construire une logique parallèle régie par le plaisir final (exemples $n^{\circ} 7$ ). 


\title{
Exemples $\mathrm{n}^{\circ} 6$
}

\author{
Entretien Tom \\ Locuteur Tom \\ Il y a un truc de... assez curieux, que je trouve, c'est que... je sais pas, j'étais \\ extrêmement collectionneur, et je ne visionnais quasiment jamais.[...] \\ À l'époque, j'étais pas un fana de la sécurité informatique, j'utilisais le peer-to-peer, \\ le... le peer-to-peer pour ce genre de trucs... C'est euh... ce qui est le plus surveillé \\ par... par la police. Donc voilà, c'est comme ça qu'ils... qu'ils m'ont repéré, et qu'à \\ six heures du mat' j'ai eu le droit à... à leur charmante visite. \\ Entretien Louis \\ Locuteur Louis \\ Et euh... la pédopornographie, ce qui s'est... ce qui s'est passé, c'est qu'en fait, elle \\ m'a... à force de... comme du bourrage de crâne, de regarder sept, huit heures, voilà, \\ par jour, t'as... t'as une vision déformée des choses.
}

\section{Exemple $n^{\circ} 7$}

\section{Entretien Thomas}

Locuteur Thomas: Les abus sexuels que j'ai subis ont permis que se fassent en moi une addiction à la sexualisation mentale puis à la masturbation très tôt très très trop tôt avant même que je sache comment ça marche en fait, en réalité quoi.

\section{Exemples $\mathrm{n}^{\circ} 8$}

\author{
Entretien Alexandre \\ Modérateur \\ Ben ça t'as jamais motivé de rencontrer le jeune que tu filmais? \\ Locuteur Alexandre \\ Euh le garçon, ben non pas'que ça restait toujours dans le domaine de l'image ! \\ Entretien Albert \\ Locuteur Albert \\ Pour moi, c'était suffisant j'avais une satisfaction de me faire passer pour une jeune \\ fille de 17 ans et de faire réagir des garçons pleins d'hormones et je trouvais mon \\ plaisir dans ce scénario, là-dedans.
}

21 Cette première section montre que, chez les participants étudiés, les outils technologiques induisent des mécanismes de prise de distance qui ne permettent plus de distinguer et de cloisonner les registres de l'affectif et du sexuel. Cette distorsion par le prisme déformant de la technologie se produit entre autres au travers de procédés lexicaux (désignation $v s$ absence de désignation du mot pivot au profit de mots sémantiquement plus vagues ou plus englobants) ou syntaxiques (tournures négatives, concessives, restrictives, oppositionnelles). Cette distanciation qui tente à désincarner l'enfant participe alors à minorer la responsabilité des auteurs dans les faits qui leur sont reprochés. Les auteurs mettent alors en place des mécanismes de défense pour justifier la distorsion qu'ils vivent avec le réel.

\section{Mise en mots des mécanismes de défense}

Les participants à ce groupe de paroles sont dans une situation de justifications ; dès lors qu'ils se racontent, ils tentent « d'expliquer, de justifier les opinions qu'on leur prête ou simplement de susciter un questionnement" (Amossy 2000) autour de leurs comportements déviants. Rappelons que se dévoiler et se raconter relève d'un domaine à risques d'où l'abondance de formes de modulation (processus tendant à diminuer la part 
de subjectivité que chacun peut investir dans l'interaction : « registres de l'euphémisme, de l'atténuation, des circonlocutions, du discours précautionneux, des lexicalisations prudentes, des actes indirects, des préliminaires, des justifications, des auto-corrections, etc. » (Vion, 1992-2000: 244) et de tension (processus inverse : « registres de l'hyperbole, de l'emportement, des lexicalisations marquées et pittoresques, des actes directs, etc.» ( ibid)).

Les échanges dans ce groupe de paroles portent donc sur le rapport entre les participants et les outils technologiques, or si l'on se réfère à l'analyse du dendrogramme ${ }^{6}$ du verbatim situé en annexe, les propos se rapportant à la technologie et au mineur ne constituent que $18,1 \%$ du corpus (classe 5), ce qui veut dire que les participants consacrent une large part (81,9\%) à évoquer ce qui ne va pas avec $29,3 \%$ du corpus (classe 4), leur sexualité (malheureuse) avec 14,8\% du corpus (classe 3), la prise en charge, les soins à prodiguer avec $24,1 \%$ du corpus (classe 2) et l'évocation de la nature de leurs addictions avec 13,6\% du corpus (classe 1$)$.

Cette photographie du verbatim montre que les mécanismes de défense ne portent pas exclusivement sur le rapport technologie / mineur, mais sur la mise en mots de conseils amicaux qui valorisent le réel pour dévaloriser l'outil.

Dans l'échange entre les participants émergent les actes directs ancrés dans l'énonciation pour exprimer le conseil qui repose sur une inférence (reconnaitre un acte déviant) pour valoriser la manière dont le participant peut le dépasser. La répétition des constructions ( si+ présent+intensifieur....+ c'est qui essentialise l'acte ou surenchères avec tournures impératives) participe tant à prodiguer du réconfort qu'à orienter le participant vers un retour dans le réel.

\section{Entretien Valentin}

Locuteur un participant : Franchement, si tu arrives vraiment à... à rechercher ça, [l'arrêt du visionnage des supports pédopornographiques] c'est une belle évolution. La seule chose, moi, qui me gêne, c'est que si tu arrives vraiment à seize ans, c'est, c'est... c'est gagné, mais intéresse-toi à l'humain. Arrête avec le virtuel. C'est-à-dire maintenant, tu es arrivé à un stade où tu n'as pas besoin de culpabiliser hein. Et donc essaye de... de nouer des contacts humains. Parce que je sais... l'ordinateur, c'est froid hein!

D'autres mécanismes de défenses apparaissent également à travers un travail important sur soi avec des prises de résolutions à l'aide d'actes volitifs qui réaffirment une intention personnelle d'agir (j'essaye de et je me suis rendu compte) faisant passer au second plan la gravité des addictions. En fin de séquence, le participant recatégorise l'énumération des addictions par un lexème sémantiquement englobant qui reprend chacune d'elles (les addictions aux jeux vidéo et au cannabis renvoient à problèmes personnels).

\section{Entretien Thomas}

Locuteur Thomas: Mais bon justement c'est pour ça que j'essaye enfin aujourd'hui, avec les cours, j'essaye justement de me débarrasser un peu de toutes mes addictions en fait. Donc euh un peu informatique, un peu des jeux vidéo, et puis pas mal de cannabis aussi, je fume beaucoup. Mais pareil : je me suis rendu compte que pour le cannabis, en fait avec le temps je me suis rendu compte que c'était simplement parce que je voulais fuir en fait mes problèmes

Un autre travail sur soi consiste à identifier ce qui reste à faire pour sortir des addictions. Le participant évalue graduellement ce qu'il ne fait plus par rapport à ce qui a été fait et ce qui reste à faire.

\section{Entretien Alain}


Locuteur Alain : Alors maintenant j'arrive à, j'arrive à à ne plus le faire, à plus trop le faire en tout cas à ne plus télécharger, ça s'est sûr. Non mais. En fait c'est comme toutes addictions, c'est comme cela, il y a des paliers, il y a un moment on arrive à dépasser un palier en se disant, ça je, ça je ne le fais plus. Après c'est un deuxième palier, ça je ne le fais plus et plus on avance dans les paliers moins on fait de chose, euh, moi voilà, je n'ai pas dépassé tous les paliers, j'en ai conscience. Il y a des moments où j'ai encore des des, j'ai encore des moments de faiblesse et ou je, je ne vais que consulter des images. Je ne télécharge plus rien, je je je ne suis pas inscrit sur quoique ce soit, je ne fais pas partie d'un forum...

Les participants tentent de mettre fin à leurs addictions qui durent pour certains depuis de nombreuses années; devant leur incapacité à les vaincre seuls et à avoir essayé d'autres dérivatifs (alcool, drogues douces dures, jeux en ligne...), ils négligent leurs protections informatiques augmentant ainsi les possibilités de se faire repérer par la police. Il y a donc un temps pour l'arrestation que certains vivent comme un moment de soulagement voire de délivrance.

\section{Entretien Albert}

Je ne sais pas encore [le fait de diffuser des documents]. J'y travaille. Je travaille dessus, j'essaie d'analyser ça mais je ne sais pas encore. Je ne sais pas si c'est pas une démarche de pouvoir, ça fait 25 ans que, oui c'est ça 25 ans que je vivais avec ça et le fait que je me sois fait prendre est peut-être la meilleure chose. C'est peut-être le seul moyen pour m'en sortir. Quand je communique avec d'autres personnes, ce qui est bien pire de toutes façons parce que les personnes qui voient ça [les films à caractère pédophile] sont des personnes qui veulent voir ça donc de toutes les façons ils ne te confirment pas que tu es un gros dégueulasse et que, et qu'il y a des moyens pour s'en sortir, non, ils te disent «t'en as pas d'autres »?

\section{Entretien Alain}

Oui j'ai fait ça, un peu, comme un gogol mais en même temps, c'est c'est presque, enfin je n'ai jamais cherché à me protéger si j'avais été plus malin ou si j'avais été plus fort en informatique, $j$ 'aurai... un système plus protégé mais j'en avais tellement mare que finalement, le matin où ils sont v'nus, j'ai pas eu peur et j'me suis dit qu'une page se tournait.

[...]

j'ai pu faire un vrai travail donc euh, lors de ma garde à vue, on m'a pris tout le matériel, j'ai vraiment réalisé que, que enfin, il y avait un gros problème, en fait, autant j'avais minimisé la première fois, autant là je me suis dit, non seulement t'es dans la merde mais il y a vraiment quelque chose qui ne va pas,

Et un temps pour la justice avec d'autres mécanismes de défense dont par exemple, celui consistant à dénigrer une catégorie de professionnels (les psychologues) pour discréditer l'ensemble de la justice en tant qu'institution. Les participants semblent unanimes pour dire que les psychologues ne sont pas bien formés aux problématiques de la pédophilie en général et de cyberpédophilie en particulier.

\section{Entretien Martial}

Ouais voilà, ça [les rendez-vous réguliers avec une psychologue] ne m'apportait plus rien. Et on avait fait le tour. Et ça servait plus à rien d'y aller. Mais j'y suis allé pendant... pendant plusieurs années, une fois par semaine au début. Et vraiment, la dernière année, on était descendus à une fois toutes les deux semaines. Parce que ça commençait déjà à devenir un peu redondant tout ce qu'on racontait. Donc je me suis dit : «Bon... voilà!» Mais effectivement, pour la justice, elle, tout ce qu'elle veut, c'est, c'est... que tu ailles voir... que t'ailles voir un professionnel, que t'ailles voir un charlatan... T'irais voir une diseuse... une diseuse de bonne aventure, ça serait pareil hein! j'ai l'impression. Si elle marque "psychologue " au lieu de «voyante », pour eux c'est la même hein. Je veux dire... i n'y connaissent rien aux 
addictions à Internet Ah non mais... Je sais pas dans quelle mesure c'est approprié quoi donc moi, faut pas m'parler de justice efficace! mais les écoutants qui officient dans les lieux d'écoute constatent une augmentation de cette consommation probablement due en partie à l'accès technologique largement facilité et simplifié. Cette étude, à partir d'un verbatim restreint, montre des tendances qui mettent en lumière des logiques argumentatives qui minimisent la gravité des actes des auteurs en invoquant la distance qu'il y a entre les outils technologiques et ce à quoi renvoient les images, c'est-à-dire à l'enfant. Cette distorsion par le prisme déformant de la technologie entraine ainsi une récusation de la dénomination de " pédophile " puisque ce ne sont que des « images ». D'autres logiques argumentatives émergent pour assurer une ligne de défense qui reposent sur l'argument technologique à chaque étape de leur récit : que ce soit pendant les nombreuses années d'utilisation des outils pour regarder (voire pour diffuser) du contenu à caractère pédopornographique ou que ce soit au moment de la confiscation du matériel lors de l'interpellation par les autorités ou ensuite, au cours de l'action de la justice.

On constate par ailleurs que l'enfant est très largement évoqué par des désignations objectives (enfant, fille, petite fille, gamin, garçon, gosse fillette, mineur avec 235 occurrences au total contre 11 occurrences seulement de l'enfant en tant que victime qui est reconnu en tant que tel par la loi. Cette différence dans la désignation, et donc de point de vue, corrobore une fois de plus la difficulté pour ces auteurs de se situer par rapport aux victimes et par rapport à leurs actes. 


\section{BIBLIOGRAPHIE}

Amossy R. (2010), La présentation de soi. Ethos et identité verbale, Puf.

Barbet D., Mayaffre D., (2007), « Débats pour l'Élysée », Mots. Les langages du politique [En ligne], 89 | 2007. URL : http://mots.revues.org/18733

Bass D., Caevel H. (2005), Au fil de la parole, des groupes pour dire dans le secteur psychosocial, Eres, Paris.

Boullier B. (2016), Sociologie du numérique

CREIS, « Proposition de programme « informatique et société numérique » pour l'option informatique au lycée », Terminal, 110 | 2012, 133-141.

Douzet F. (2014), « La géopolitique pour comprendre le cyberespace », La Découverte |

« Hérodote » 2014/1 n 152-153, 3-21.

Fassin D. (2004), Des maux indicibles. Sociologie des lieux d'écoute, La Découverte, Paris.

Lebart L. \& Salem A. (1994), Statistique textuelle, Broché, Paris.

Longhi J. (2015), «Stabilité et instabilité dans la production du sens : la nomination en discours », Langue française, vol. 188, no. 4, 2015, 5-14

Maingueneau D. (1991), L'Analyse du discours, Introduction aux lectures de l'archive, Paris, Hachette, Nouvelle édition mise à jour : l'Analyse du discours, Hachette.

Nossik S. 2011, «Les récits de vie comme corpus sociolinguistique : une approche discursive et interactionnelle », Corpus, 10 , 2011, 119-135.

Nossik S. 2014. Approches discursives des récits de vie Semen, numéro 37.

Paveau M.-A. (2014), Le discours pornographique, éditions de la musardine, collection « l'attrapecorps ", $400 \mathrm{p}$.

Paveau M.-A. \& Perea F. (coord.). (2015). « Corpus sensibles », cahiers de praxématique, $\mathrm{n}^{\circ} 59$.

Perelman Ch. \& Olbrechts-Tyteca L. (1958). La nouvelle rhétorique ; Traité de l'Argumentation (Coll. Logos). Presses Universitaires de France, Paris.

Plantin C. (2016), Dictionnaire de l'argumentation, une introduction aux études d'argumentation, Ens Edition, Paris.

Siblot P. 1998, « De la dénomination à la nomination », cahiers de praxématique [en ligne], 36 | 2001, document 8,1/1/2009. URL : http://praxematique.revues.org/368

Vion R. (1994), «L'analyse des interactions verbales », Les Carnets du Cediscor [En ligne], 4 | mis en ligne le 22 juillet 2009, consulté le 16 janvier 2017. URL : http://cediscor.revues.org/349.

Zetlaoui T. (2016), Société numérique

\section{ANNEXES}

Figure 3 : Répartition des éléments techniques 


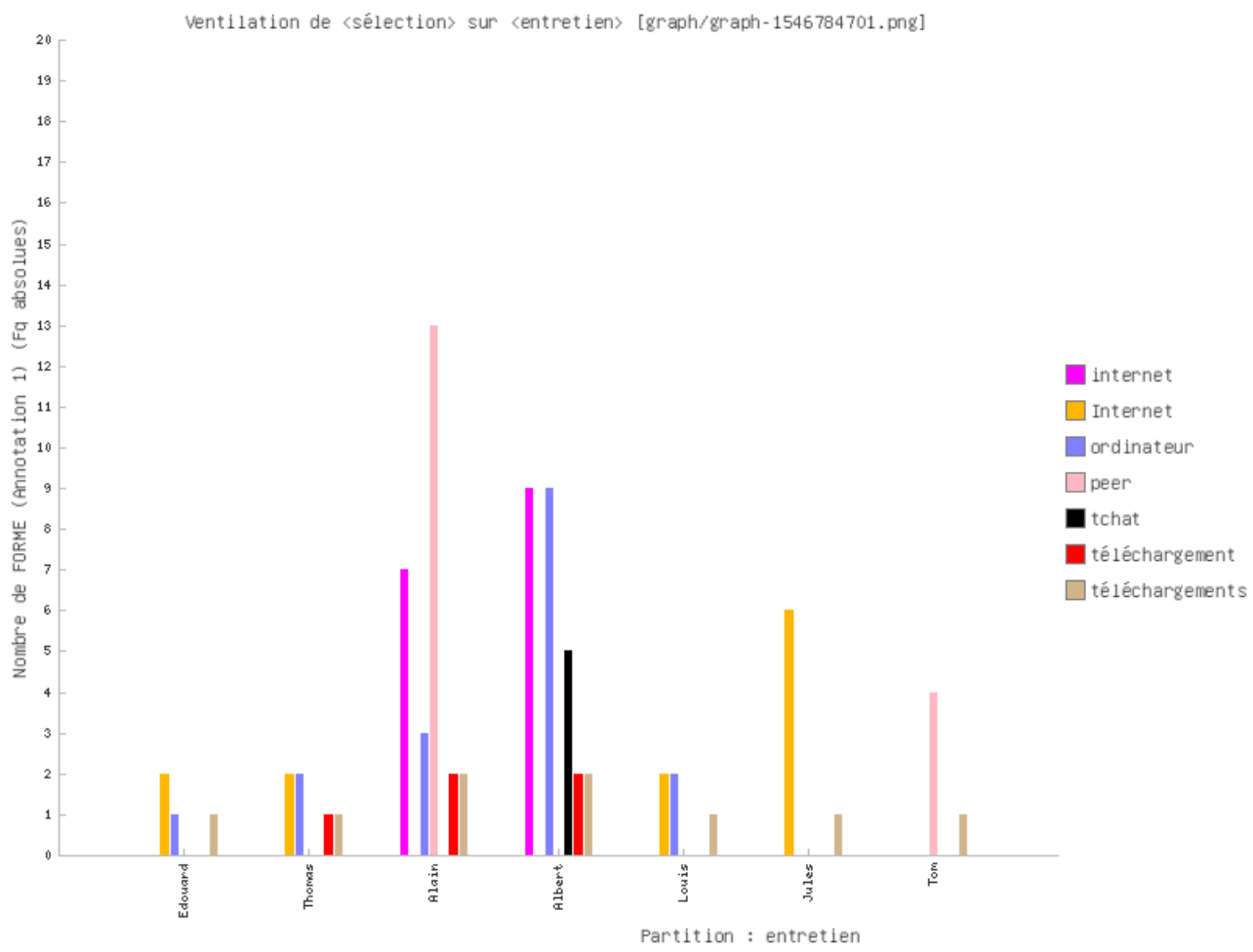

Figure 4 : Dendrogramme du verbatim
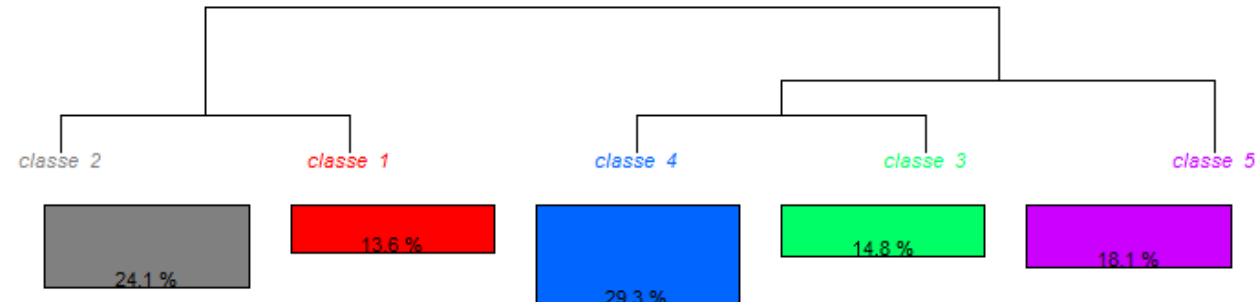

premier
voir
moment
parole

locuteur
martial
boire
arrêter
continuer
rendre
semaine
drogue
bouteille
compte
cannabis
film
nouveau
finalement
psychistre
jardin
persusder
face
caquer
sbri
whisky
matéo
fumer
temps
travail
finir
fantasme

enfant
question
poser
comprendre
place
couple
problème
femme
rapport
marier
absolument
pédophile
relation
mère
peur
vachement
cairement
analyse
côté
mettre
toucher
remettre
chose
famille
vincent
tête
arave
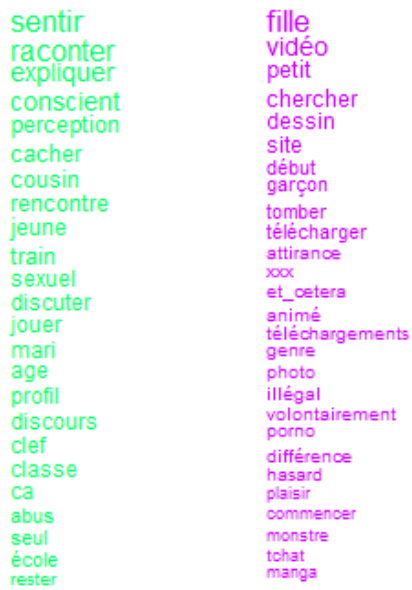

\section{NOTES}

1. En 1958, Perelman et Olbrechts-Tyteca ont proposé la définition suivante de l'argumentation : «L'objet de la théorie de l'argumentation est l'étude des techniques discursives permettant de 
provoquer ou d'accroître l'adhésion des esprits aux thèses qu'on présente à leur assentiment » (1958:5).

2. Cette perspective élargie de l'argumentation va au-delà de l'opposition de deux thèses antagonistes ou dans une définition de l'argumentation rhétorique qui vise « une entreprise de persuasion sociale par le discours L'art de la rhétorique n'est-il pas l'art d'avoir de l'influence sur les âmes par le moyen du discours prononcé non seulement dans les tribunaux et dans toutes les autres assemblées mais aussi dans les réunions privées" (Platon, Phèdre 143-144). En effet, la production de paroles de ces locuteurs ne vise en rien à nous convaincre qu'ils sont innocents ou coupables, elle n'a pas davantage l'objectif de nous persuader qu'ils disent la vérité.

3. Les autres participants à ce groupe de paroles sont des victimes, des agresseurs condamnés, en cours de jugement ou ayant déjà purgé une peine et des pédophiles dits « abstinents ».

4. Le prénom est remplacé par un autre prénom portant la même initiale, nous avons également respecté le genre.

5. Rappelons que certains participants au groupe de paroles sont des détenteurs et des diffuseurs de vidéos pédopornographiques sur internet et d'autres sont des victimes, des agresseurs...

6. Le dendrogramme est une classification ascendante hiérarchique réalisée à partir du mode de calcul Reynaert avec le logiciel Iramuteq. On constate ici que les 5 classes regroupent d'un côté 3 classes (classes 3, 4 et 5) qui totalisent 62,3\% du verbatim et de l'autre, 2 classes (classes 1 et 2) qui totalisent $37,7 \%$ du verbatim.

7. Les autres participants peuvent-être des victimes, des agresseurs....

INDEX

Mots-clés : adolescents, diffusion, possession, vidéos pédopornographiques

Keywords : teenagers, distribution, possession, child pornography videos

\section{AUTEUR}

\section{FRÉDÉRIC PUGNIERE-SAAVEDRA}

maître de conférences en sciences du langage, Université de Bretagne Sud, frederic.pugnieresaavedra@univ-ubs.fr 